Cita bibliográfica: Salvador Oliván, J. A., y Escolano Utrilla, S. (2018). Comparación de indicadores bibliométricos en las revistas de geografía indizadas en Web of Science. Boletín de la Asociación de Geógrafos Españoles, 76, 131-152. doi: 10.21138/bage.2518

\title{
Comparación de indicadores bibliométricos en las revistas de geografía indizadas en Web of Science
}

\author{
Comparison of bibliometric indicators \\ in journals of geography indexed in Web of Science
}

\author{
José Antonio Salvador Oliván \\ jaso@unizar.es \\ Departamento de Ciencias de la Documentación e Historia de la Ciencia \\ Universidad de Zaragoza (España)
}

\author{
Severino Escolano Utrilla \\ severino@unizar.es \\ Departamento de Geografía y Ordenación del Territorio \\ Universidad de Zaragoza (España)
}

\section{Resumen}

La estimación de la calidad de las revistas científicas, necesaria para diversos propósitos, se realiza habitualmente a través de indicadores bibliométricos. En este trabajo se estudia el comportamiento de estos indicadores para las revistas de las categorías Geografía y Geografía Física de Web of Science en 2015. Los resultados muestran que las revistas de Geografía Física tienen mayor impacto que las de Geografía y que los indicadores presentan un alto grado de correlación y de concordancia en la clasificación de las revistas en cuartiles, siendo ocupados los primeros lugares por las mismas revistas. Para evaluar la influencia de las revistas de Geografía en la comunidad científica, es recomendable utilizar indicadores complementarios a los habituales que permitan obtener una visión más completa de su impacto. 
Palabras clave: indicadores bibliométricos; revistas de geografía; factores de impacto; índices de concordancia; coeficientes de correlación.

\begin{abstract}
The quality of scientific journals, required for various purposes, is usually estimated by bibliometric indicators. This study investigates the behavior of these indicators related to journals classified as Geography and Physical Geography in Web of Science (year 2015). Results show that the impact of Physical Geography journals is greater than the Geography journals. In addition, there is an important degree of correlation and concordance in the classification in quartiles: same journals are in the top of the ranking in both categories. In order to improve the process of assessment of Geography journals by the scientific community, we propose the use of supplementary indicators to provide a complete perspective.
\end{abstract}

Key words: bibliometric indicators; geography journals; impact factors; concordance indices; correlation coeficients.

Agradecimientos: Grupo de investigación GEOT (Grupo de Estudios en Ordenación del Territorio). Instituto Universitario de Ciencias Ambientales (IUCA). Proyecto: 2014/0032: Acuerdo de colaboración entre Ebrópolis y la Universidad de Zaragoza para la realización de actividades de investigación. en la portada tras las palabras clave y recogerá los datos principales del proyecto o los proyectos que lo sustentan.

\title{
1 Introducción
}

Las revistas son el medio por excelencia utilizado por los científicos para la difusión y comunicación de los resultados de una investigación. Para los investigadores, la elección de la revista en la que publicar es una decisión importante que se toma en función de diversos criterios como el impacto, prestigio, influencia, calidad o visibilidad que tiene en su disciplina y otros.

Dos son los métodos empleados habitualmente para evaluar la calidad de las publicaciones, el análisis realizado por expertos y el recuento de citas que reciben (Sellers et al.., 2004). Dado el carácter subjetivo y laborioso de la evaluación por expertos, se han propuesto distintos indicadores que no miden directamente la calidad pero que la estiman de manera aproximada a través del impacto en la comunidad científica determinado por el número de citas que cada revista recibe (Setti, 2013). Aunque se ha encontrado una correlación positiva entre la tasa de citación y la calidad determinada por expertos (Richards et al., 2009), el número de citas depende también de los hábitos de publicación en un campo científico y de la popularidad del tema. 
Los indicadores se han clasificado en aquellos que miden la popularidad, que tienen en cuenta el número absoluto de citas recibidas por los artículos publicados, y en los que miden el prestigio de las revistas, denominados así porque ponderan las citas según la fuente de la que procedan, asignando más peso a las citas de aquellas revistas que son más citadas (Bollen et al., 2006; Franceschet, 2010).

El primer indicador bibliométrico creado fue el Factor de Impacto (FI), propuesto en 1955 por Eugene Garfield para medir la importancia relativa de una revista en un determinado campo (Garfield, 2006). El FI de las revistas se publica anualmente en el Journal Citation Reports (JCR) y se define como el número de citas que reciben durante los dos años anteriores los ítems publicables en esos dos años; para su cálculo solo se utilizan las revistas indizadas en la base de datos Web of Science (WoS) de Thomson Reuters.

En la actualidad, el FI goza de una amplia aceptación en el mundo científico y es utilizado como índice de calidad de las revistas por las comisiones de evaluación de las instituciones académicas y de investigación para seleccionar al personal y para evaluar la calidad de las publicaciones de su personal. Además, para que los profesores e investigadores en España puedan obtener una evaluación positiva de tramos de investigación se requiere, en la mayoría de las áreas de conocimiento, que los artículos estén publicados en revistas situadas en el primer, segundo o tercer cuartil de las categorías científicas del JCR (dependiendo del área). En consecuencia, día a día crece la presión sobre los investigadores para publicar en revistas de alto impacto (Bosman, 2009). Estas revistas, tal y como se ha evidenciado en un estudio realizado por Huan (2016), son las que publican más artículos, algo que puede deberse a que atraen un gran número de originales enviados para ser sometidos a evaluación por la gran competencia existente entre los investigadores para publicar en ellas, y al proceso severo de revisión para seleccionar aquellos de mayor calidad. Por otra parte, también las editoriales y los directores de bibliotecas recurren a este indicador, en el primer caso para atraer manuscritos de investigadores y conocer la posición de sus revistas con respecto a la competencia, y en el segundo para cancelar, renovar o solicitar nuevas suscripciones de revistas.

Sin embargo, el Fl ha recibido muchas críticas por sus limitaciones; entre las descritas en la bibliografía destacan: todas las citas tienen el mismo peso y no se tiene en cuenta el prestigio de las revistas citantes, el periodo de 2 años se considera insuficiente, falta de normalización por los hábitos de publicación y de citación en los diferentes campos y disciplinas, sesgo a favor de las revistas que publican artículos de revisión, distribución asimétrica del número de citas recibidas y la influencia de factores que no tienen ninguna relación con la calidad de las revistas (Seglen, 1997; Glanzel y Moed, 2002; Leydesdorff, 2008; Pendlebury, 2009; Archambault y Lariviere, 2009; Vanclay, 2012; Mutz y Daniel, 2012). 
Para paliar las limitaciones del FI, se han desarrollado otros indicadores bibliométricos alternativos para evaluar la influencia de una revista científica. Entre los indicadores de popularidad se encuentran el Factor de Impacto de 5 años (FI5), el Impact Per Publication (IPP) y el Source Normalized Impact per Paper (SNIP). Los indicadores de prestigio, Eigenfactor, Article Influence Score (AIS) y SCImago Journal Rank (SJR) se inspiran en el algoritmo PageRank de Google como esquema de ponderación de las revistas citantes.

A partir de los indicadores mencionados se han propuesto diversas variantes que no se tratarán en este estudio ya que muchos de ellos proporcionan esencialmente la misma información y, como recomienda Waltman (2016) en una revisión reciente sobre los indicadores de impacto, no se deberían introducir nuevos indicadores al menos que ofrezcan un valor añadido a los ya existentes.

Habida cuenta de la importancia que adquieren los indicadores de calidad e impacto en la difusión del conocimiento científico (y tal vez en la praxis de algunos científicos) y la ausencia de un único indicador apropiado para valorar la calidad de la producción científica en todas las circunstancias (Bar-lan, 2012), es muy conveniente estudiar el comportamiento de tales indicadores en el contexto de cada disciplina para conocer cuáles son fundamentales y cuáles aportan información complementaria, ya que es bien conocido que los factores de impacto de las revistas difieren según las disciplinas a las que pertenecen, siendo debidas estas diferencias a distintas prácticas de citación, al lapso de tiempo entre la publicación de un artículo y su posterior cita (Althouse et al., 2009).

En la bibliografía especializada se han llevado a cabo varias investigaciones en las que se comparan algunos de estos indicadores en diferentes disciplinas científicas, sobre todo el FI, SJR, AIS e índice $H$, pero no se ha encontrado ninguno en Geografía. Los estudios publicados se han centrado exclusivamente en el Fl, como el realizado por Barriocanal y Copete (2005) en el que se analiza la evolución del FI en las revistas de Geografía durante el periodo de 1989-2002, y algunos otros sobre el uso de las citas de un área concreta de Geografía y su relación con otros campos de conocimiento (Rholi y Kam-Biu, 2008; Bosman, 2009; Laffan, 2010; Scarletto, 2014).

Con este contexto se aborda aquí el estudio de los indicadores bibliométricos de popularidad y de prestigio calculados a partir de las bases de datos Web of Science y Scopus en las revistas de Geografía incluidas en las categorías temáticas de "Geografía" y "Geografía Física" del Journal Citation Reports (JCR) con los siguientes objetivos:

- Contrastar si existen diferencias en el impacto y prestigio de las revistas de las dos categorías temáticas.

- Analizar el grado de correlación entre los indicadores bibliométricos para determinar cuáles son redundantes desde el punto de vista estadístico y cuáles aportan información complementaria. 
- Valorar el grado de concordancia entre los indicadores bibliométricos al clasificar las revistas en los distintos cuartiles.

- Identificar las diferencias en los rangos de las revistas situadas en el primer cuartil de FI creados con los distintos indicadores bibliométricos.

\section{Método}

La población de este estudio la componen todas las revistas de la disciplina Geografía indizadas en WOS en el año 2014 y clasificadas en las categorías "Geografía" (Social Science Citation Index) y "Geografía Física" (Science Citation Index) del JCR. Según el JCR, la categoría Geografía Física abarca aquellos recursos que tratan de la diferenciación de las áreas de la superficie terrestre mostradas en el carácter, disposición e interrelaciones en el mundo de elementos como el clima, altitud, vegetación, población, uso del suelo e industrias, así como las áreas unitarias formadas por estos elementos individuales. La categoría de Geografía comprende aquellos recursos relacionados con los aspectos socio-culturales de la superficie terrestre centrados en los aspectos humanos, económicos, políticos, urbanos y medioambientales de la disciplina, incluyendo la historia de la geografía y la cartografía.

Aunque la clasificación precedente no refleja bien las diferencias de los contenidos de las publicaciones, se ha mantenido porque de ellas se dispone de series de datos coherentes. La categoría Geografía abarca, aproximadamente, los contenidos de la categoría "Geografía Humana"

Para la identificación y selección de las revistas se utilizó la herramienta InCitesTM Journal Citation Reports (https://jcr.incites.thomsonreuters.com). El número total de revistas seleccionadas fue de 116, distribuidas de la siguiente manera: 76 de Geografía y 46 de Geografía Física. De ellas, 6 están clasificadas en las dos categorías: Landscape and Urban Planning, Erde, Erdkunde, International Journal of Geographical Information Science, Journal of Maps y Revista de Geografía Norte Grande.

Los datos de los indicadores bibliométricos de las revistas se obtuvieron en enero de 2016 y corresponden al año 2014 (último año calculado y accesible del JCR en esa fecha): Factor de impacto, Factor de impacto 5 años, e Influencia del artículo. El Eigenfactor se desechó ya que no está normalizado por el número de artículos. Los indicadores se definen como:

El Factor de impacto (FI) de una revista es el cociente entre el número de citas que reciben durante los dos años anteriores los ítems publicables en esos dos años (Garfield, 2006).

El Factor de impacto de 5 años (FI5) se calcula de la misma manera pero ampliando la ventana temporal del número de citas recibidas e ítems publicables a 5 años. 
Influencia del Artículo (Article Influence Score, AIS): El numerador de la fórmula equivale al indicador Eigenfactor, que se caracteriza por excluir las autocitas, analizar un periodo de 5 años, tener en cuenta las citas recibidas en revistas y otras publicaciones procedentes de Ciencias Sociales y Ciencia, y también las diferencias en el impacto entre las disciplinas científicas, dando más peso a aquellas citas que proceden de artículos publicados en revistas que son a su vez más citadas (Bergstrom, 2007). Al dividirlo por el número de artículos publicados, mide la influencia media de los artículos de una revista, siendo comparable conceptualmente al Factor de Impacto de 5 años, con la diferencia de que las citas reciben un peso diferente según la influencia de las revistas citantes (Bergstrom et al., 2008).

Desde SClmago Journal \& Country Rank (http://www.scimagojr.com/journalrank.php) se descargaron los datos de todas las revistas de Scopus y los indicadores Scimago Journal Rank, índice $H$, y citas recibidas/documentos publicados en los dos años anteriores. Los indicadores se definen de la siguiente manera:

Scimago Journal Rank (SJR): Mide la influencia o el prestigio de las revistas. A diferencia del Fl, amplía la ventana de citación a 3 años, otorga más peso a las citas procedentes de revistas con mayor prestigio (aquellas que, a su vez, reciben más citas), limita el número de autocitas y tiene en cuenta en el denominador todos los documentos publicados en la revista (González-Pereira et al., 2010).

Índice H: Fue propuesto inicialmente por Hirsch para cuantificar la producción científica de un investigador, y se define como el número $h$ de artículos con un número de citas $\geq h$ (Hirsch, 2005). En 2006, Braun propuso aplicar este índice a la evaluación de las revistas (Braun et al., 2006).

Factor de impacto de Scopus (FI2SC): Este indicador ha sido calculado por los autores con los datos del número de citas recibidas y número de documentos publicados en una revista durante los dos años anteriores, pero exclusivamente con los datos de Scopus. Hay que indicar que difiere del FI del JCR ya que en este el denominador solo tiene en cuenta los artículos originales y de revisión, mientras que en Scopus el número de documentos publicados incluye todos los tipos publicados en una revista.

Desde Journal Metrics (http://www.journalmetrics.com/) se descargaron las revistas de Scopus y los indicadores IPP y SNIP. Los indicadores se definen de la siguiente manera:

Impact Per Publication (IPP): Se calcula dividiendo el número de citas recibidas por los artículos publicados en una revista durante los tres años anteriores entre el número de artículos publicados en esa revista durante esos tres años (Elsevier, 2016). 
Source Normalized Impact per Paper (SNIP): Desarrollado por Henk Moed en la Universidad de Leiden, es el IPP normalizado: el numerador contiene el IPP y el denominador el potencial de citación en la disciplina de la revista teniendo en cuenta las características y diferencias que existen entre las disciplinas como la frecuencia con la que se citan los autores, la rapidez de maduración del impacto de las citas y el grado de cobertura de la base de datos para un campo concreto (Moed, 2010). En otras palabras, mide el impacto de las citas teniendo en cuenta el contexto y proporciona una corrección según las prácticas de citación en campos científicos, obteniendo valores más bajos cuando la tasa de citación es muy alta, y viceversa.

El análisis estadístico se realizó con el programa SPSS v.22. El análisis de los factores de impacto y de prestigio en las dos categorías temáticas se llevó a cabo con el test t de Student, aplicando en el caso de incumplimiento de homogeneidad de varianzas el test de Welch. La correlación entre los indicadores se analizó con el coeficiente de correlación de Spearman. Las revistas se clasificaron en cuartiles en cada uno de los indicadores y se analizó la concordancia de esta clasificación con el índice de Kappa ponderado (Cohen, 1968), en el que se asignan unos pesos para cuantificar la importancia relativa de los desacuerdos, ya que no es lo mismo que haya una discrepancia de clasificar a una revista en un primer a un segundo cuartil, que entre un primer cuartil y un cuarto cuartil; por lo tanto, estos pesos ajustan el grado de discordancia. Se han aplicado pesos lineales (Cichetti y Allison, 1971). Para la interpretación de la fuerza del coeficiente de Kappa se sigue la clasificación de Landis y Koch (1977): débil para valores menores a 0,40, moderada entre 0,41 y 0,60, buena entre 0,61 y 0,80, muy buena para valores superiores a 0,80 hasta 1 .

\section{Resultados}

Los datos de todos los indicadores presentan una gran dispersión y una distribución muy asimétrica positiva y leptocúrtica, salvo el índice H y SNIP en Geografía Física que siguen una distribución platicúrtica. La condición de normalidad, contrastada con el test de Kolmogorov-Smirnov, no se cumple en ningún indicador cuando se contempla toda la disciplina ni en la categoría de Geografía, por lo que para evaluar la correlación se ha aplicado el coeficiente no paramétrico de Spearman.

\subsection{Nivel de los indicadores en las categorías Geografía y Geografía Física}

El impacto bruto de las citas obtenidas por los artículos publicados en las revistas de Geografía Física es superior en todos los indicadores al obtenido en las revistas de Geografía, tal y como se observa en la Tabla 1 y en la Figura 1 al analizar el FI, FI5, FI de Scopus, el IPP y el índice H. Estos resultados coinciden con los obtenidos por los estudios de Rohli y Kam-Biu (2008) y García Ruiz 
(2008) cuando concluye que los artículos españoles de geografía más citados en revistas internacionales corresponden al campo de Geografía Física.

Tabla 1. Comparación del nivel de los indicadores bibliométricos entre las categorías Geografía y Geografía Física

\begin{tabular}{|c|c|c|c|c|}
\hline \multirow{2}{*}{ Indicador } & \multicolumn{2}{|c|}{ Media } & $\begin{array}{c}\text { Diferencia } \\
\text { medias (IC 95\%) }\end{array}$ & $p$ \\
\cline { 2 - 3 } & Geografía & Geografía Física & $0,379-1,309$ & 0,001 \\
\hline FI & 1,283 & 2,127 & $0,283-1,343$ & 0,003 \\
\hline FI5 & 1,689 & 2,502 & 0,079 \\
\hline AIS & 0,637 & 0,825 & & 0,128 \\
\hline SJR & 0,886 & 1,111 & & 0,004 \\
\hline FI2SC & 1,437 & 2,143 & $0,238-1,174$ & 0,866 \\
\hline SNIP & 1,132 & 1,151 & & 0,001 \\
\hline IPP & 1,393 & 2,226 & $0,364-1,302$ & 0,005 \\
\hline Índice H & 35,61 & 50,87 & $4,674-$ & 0,854 \\
\hline
\end{tabular}

Leyenda: FI: Factor de Impacto; FI5; Factor de Impacto 5 años; AIS: Article Influence Score; SJR: Scimago Journal Rank; FI2SC: Factor de Impacto de 2 años creado de Scopus; SNIP: Source Normalized Impact per Paper; IPP: Impact per Publication.

Fuente: elaboración propia a partir de datos del Journal Citation Reports (2016), Scimago Journal Rank (2016) y Journal Metrics (2016)

Figura 1. Valores medios de algunos indicadores bibliométricos en las categorías Geografía y Geografía Física

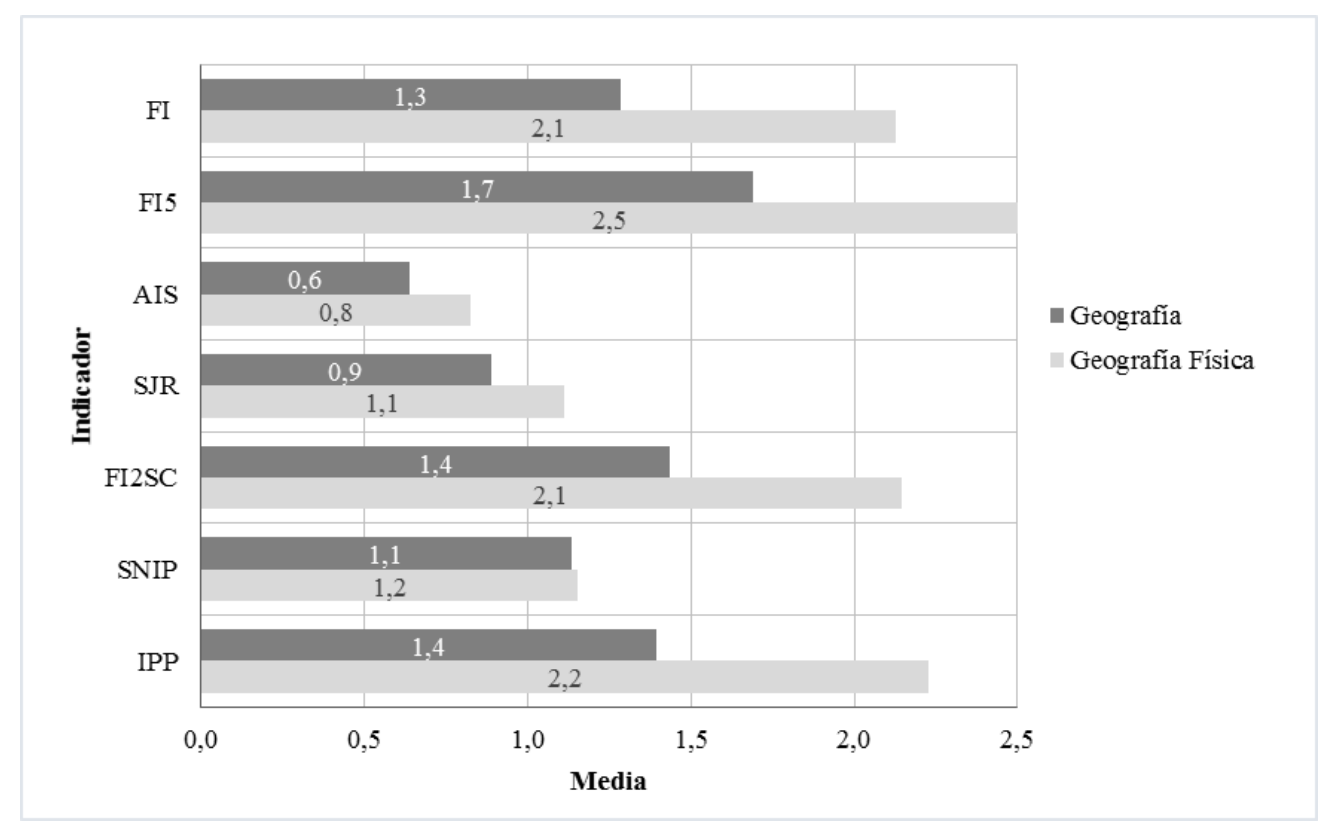

Fuente: elaboración propia a partir de datos del Journal Citation Reports (2016), Scimago Journal Rank (2016) y Journal Metrics (2016) 
La diferencia puede explicarse porque, como indica Laffan (2010), las revistas de Geografía Física son más importadoras de citas mientras que las de Geografía Humana son más exportadoras, debido a diversos factores como pueden ser los hábitos de publicación de los investigadores. Otra razón puede atribuirse a que las áreas temáticas que publican, preferentemente en la categoría Geografía (población, espacios urbanos y rurales, ...) son multidisciplinares y, como tales, es posible que parte de la investigación en estas áreas se publique en revistas pertenecientes a otras áreas de conocimiento e incluidas en otras categorías JCR, derivándose de esta manera las citas recibidas a otras áreas. También se ha observado que los trabajos de Geografía Física suelen tener mayor influencia fuera de la disciplina de geografía (Bodman, 1991) y, por lo tanto, son citados no solo en las revistas propias de geografía sino también en las que pertenecen a otros campos científicos (meteorología, medio ambiente, geología,...), aumentando así la tasa de citación.

Se han identificado otros factores en estudios realizados en áreas científicas como medicina de urgencias (Callaham et al., 2001) y que pueden aplicarse a nuestro estudio. En primer lugar, las disciplinas con mayor tasa de citación y mayor impacto suelen tener mayor número de investigadores. Las tasas de citas, y por tanto, los factores de impacto serán mayores en aquellas áreas con mayor número de autores, salvo que se compense porque las de menor tamaño publiquen artículos con mayor número de referencias bibliográficas (Richards et al., 2009). En segundo lugar, el enfoque tan específico de las revistas puede llevar a los investigadores a publicar sus resultados en revistas clasificadas en otras áreas que tengan mayor audiencia.

El número de artículos es también un factor a tener en cuenta, ya que cuanto mayor sea el número de artículos mayor será también el número de referencias bibliográficas y, en consecuencia, la probabilidad de que un trabajo sea citado será mayor. En la categoría de Geografía Física el número medio de ítems citables (artículos publicados) es mucho mayor que en las revistas de la categoría de Geografía, a pesar de que el número de revistas es menor (Tabla 2).

\section{Tabla 2. Items citables en las categorías de Geografía y Geografía Física en las bases de datos WoS y Scopus}

\begin{tabular}{|c|c|c|c|c|c|}
\hline & $N^{\circ}$ & Media & IC 95\% & $p$ & Diferencia medias (IC 95\%) \\
\hline Items citables WoS & 76 & 51,59 & $\begin{array}{c}41,45-61,73 \\
80,72-157,32\end{array}$ & 0,001 & $27,919-106,941$ \\
\hline $\begin{array}{c}\text { Geografía } \\
\text { Geografía Física }\end{array}$ & 46 & 119,02 & \\
\hline Geografía & 76 & 140,14 & $\begin{array}{c}114,63- \\
165,66 \\
217,78- \\
412,96\end{array}$ & 0,001 & $74,623-275,826$ \\
Geografía Física & 46 & 315,37 & & \\
\hline
\end{tabular}

Fuente: elaboración propia a partir de datos

del Journal Citation Reports (2016) y Scimago Journal Rank (2016) 
Finalmente, también cabe sugerir que los procesos estudiados por los investigadores de la Geografía Física son más generalizables mientras que los procesos socioeconómicos, estudiados por la Geografía (Humana), tienen un carácter más local e idiosincrático.

De manera general, es un hecho conocido que existen diferencias importantes en los hábitos de publicación y de citación entre los investigadores de ciencias y de ciencias sociales. Para estos últimos, los libros y monografías sigue siendo un canal de comunicación importante de los resultados de sus estudios, que al no estar indizados en las bases de datos, restan citas e impacto a los artículos publicados en revistas de dicha área (Nederhof, 2006). Por lo tanto, la clasificación del JCR de las revistas de la categoría Geografía en la macroárea de Ciencias Sociales y las de Geografía Física en la de Ciencias, es otro factor que contribuye a entender la diferencia de impacto entre estas dos categorías.

En cuanto a los indicadores que ponderan las citas según la influencia de las revistas citantes (como el SJR y el AIS) o que tienen en cuenta diversos aspectos diferenciadores entre disciplinas, como el SNIP, no existen diferencias significativas entre ambas categorías.

\subsection{Correlaciones entre los indicadores}

En términos generales, la correlación entre los indicadores es muy elevada en ambas categorías de análisis, tal y como muestran los valores del coeficiente de correlación recogidos en la Tabla 3. Las tendencias más destacadas que se desprenden son las siguientes:

Los indicadores de popularidad FI, FI de 5 años, FI de Scopus e IPP miden el impacto bruto de las citas, diferenciándose en el número de años utilizado en la fórmula y en la base de datos utilizada. Se observa un grado de correlación muy alto $(>0,95)$ entre todos ellos, tanto en toda la disciplina como en las categorías JCR, lo que induce a pensar que ni el número de años ni la base de datos utilizada son factores que influyan de manera importante en un comportamiento diferente entre ellos y que prácticamente están midiendo lo mismo.

Tabla 3. Coeficientes de correlación (rho de Spearman) entre cada par de indicadores en toda la disciplina de Geografía (116 revistas) y en las categorías JCR de Geografía (76 revistas) y Geografía Física (46 revistas) (en este orden en la tabla)

\begin{tabular}{|c|c|c|c|c|c|c|c|c|c|}
\hline & \multicolumn{3}{|c|}{ WoS } & \multicolumn{5}{|c|}{ Scopus } \\
\hline & & $\mathrm{FI}$ & $\mathrm{Fl5}$ & AIS & SJR & FI2SC & SNIP & IPP & $\mathrm{H}$ \\
\hline \multirow{3}{*}{ WoS } & $\mathrm{FI}$ & 1 & & & & & & & \\
\hline & $\mathrm{Fl} 5$ & $\begin{array}{l}0,964 \\
0,953 \\
0,948\end{array}$ & 1 & & & & & & \\
\hline & AIS & $\begin{array}{l}0,909 \\
0,914 \\
0,901\end{array}$ & $\begin{array}{l}0,955 \\
0,966 \\
0,950\end{array}$ & 1 & & & & & \\
\hline
\end{tabular}


Tabla 3. Continuación

\begin{tabular}{|c|c|c|c|c|c|c|c|c|c|}
\hline & \multicolumn{3}{|c|}{ WoS } & \multicolumn{5}{|c|}{ Scopus } \\
\hline & & $\mathrm{Fl}$ & $\mathrm{Fl5}$ & AIS & SJR & FI2SC & SNIP & IPP & $\mathrm{H}$ \\
\hline \multirow{5}{*}{ Scopus } & SJR & $\begin{array}{l}0,901 \\
0,923 \\
0,880\end{array}$ & $\begin{array}{l}0,922 \\
0,944 \\
0,894\end{array}$ & $\begin{array}{l}0,919 \\
0,935 \\
0,881\end{array}$ & 1 & & & & \\
\hline & $\mathrm{F} \mid 2 \mathrm{SC}$ & $\begin{array}{l}0,970 \\
0,964 \\
0,980\end{array}$ & $\begin{array}{l}0,952 \\
0,942 \\
0,957\end{array}$ & $\begin{array}{l}0,902 \\
0,904 \\
0,900\end{array}$ & $\begin{array}{l}0,915 \\
0,918 \\
0,910\end{array}$ & 1 & & & \\
\hline & SNIP & $\begin{array}{l}0,764 \\
0,840 \\
0,720\end{array}$ & $\begin{array}{l}0,808 \\
0,876 \\
0,766\end{array}$ & $\begin{array}{l}0,789 \\
0,870 \\
0,657\end{array}$ & $\begin{array}{l}0,838 \\
0,837 \\
0,824\end{array}$ & $\begin{array}{l}0,806 \\
0,839 \\
0,782\end{array}$ & 1 & & \\
\hline & IPP & $\begin{array}{l}0,961 \\
0,959 \\
0,943\end{array}$ & $\begin{array}{l}0,971 \\
0,969 \\
0,970\end{array}$ & $\begin{array}{l}0,912 \\
0,929 \\
0,902\end{array}$ & $\begin{array}{l}0,933 \\
0,945 \\
0,926\end{array}$ & $\begin{array}{l}0,975 \\
0,969 \\
0,968\end{array}$ & $\begin{array}{l}0,824 \\
0,853 \\
0,820\end{array}$ & 1 & \\
\hline & $\mathrm{H}$ & $\begin{array}{l}0,805 \\
0,870 \\
0,673\end{array}$ & $\begin{array}{l}0,821 \\
0,890 \\
0,712\end{array}$ & $\begin{array}{l}0,824 \\
0,878 \\
0,727\end{array}$ & $\begin{array}{l}0,815 \\
0,889 \\
0,684\end{array}$ & $\begin{array}{l}0,800 \\
0,861 \\
0,684\end{array}$ & $\begin{array}{l}0,670 \\
0,793 \\
0,514\end{array}$ & $\begin{array}{l}0,811 \\
0,876 \\
0,702\end{array}$ & 1 \\
\hline
\end{tabular}

Fuente: elaboración propia a partir de datos del Journal Citation Reports (2016), Scimago Journal Rank (2016) y Journal Metrics (2016)

La ampliación de la ventana de citación en los indicadores pretende favorecer a las áreas en las que se tarda más tiempo en citar, característica propia de las ciencias sociales. En este caso, los resultados indican que en la disciplina de Geografía las revistas con mayor impacto a los 2 años, también lo son a los 3 y a los 5 años, y que no existen diferencias entre las revistas clasificadas en las categorías de Geografía Física (ciencias) y Geografía (ciencias sociales).

De la misma manera, aunque WoS y Scopus tienen diferente cobertura en cuanto a número de revistas, el rango obtenido por estas no se ve influido por la fuente de datos que se utilice. De hecho, la correlación entre el FI de WoS y el FI de Scopus, que solo se diferencian por la base de datos y los ítems citables, obtiene el máximo valor de todos los análisis por parejas (>0,96 en las tres situaciones)

Los indicadores de prestigio presentan una correlación muy alta entre el AIS y SJR, aunque algo menor en la categoría de Geografía Física $(0,881)$ que en la de Geografía $(0,935)$. Estos indicadores comparten en sus fórmulas variables como la limitación o exclusión de las autocitas y la ponderación de las revistas según su importancia, si bien se calculan a partir de bases de datos diferentes. Presentan a su vez correlaciones muy altas con todos los indicadores de impacto (> 0,90 en todos los casos) especialmente entre AIS y FI5 que comparten la misma ventana temporal de 5 años, en consonancia con el valor de 0,9626 obtenido por Yin (2011) en revistas de ingeniería química. Las diferencias son tan leves que invitan a pensar que en la disciplina de Geografía y en las dos categorías JCR, estos indicadores se comportan de manera muy similar.

La correlación entre SJR y FI es también alta, ligeramente superior a 0,90 en la categoría de Geografía y algo más baja en la de Geografía Física $(0,880)$. Estos resultados no difieren de los 
obtenidos en otros estudios realizados, por ejemplo, en revistas del sistema respiratorio donde se encontró un coeficiente r=0,94 (García Pachón y Arencibia, 2014) o en revistas de educación con un valor de $r=0,91$ (Chou, 2013). Parecidos valores se obtienen entre el SJR y el FI de Scopus y SJR, en consonancia con los resultados de Bosman (2009), que encontró un coeficiente de 0,919.

Otros indicadores: El indicador SNIP obtiene valores por debajo de 0,90 tanto con los indicadores de impacto como con los de prestigio, siendo ligeramente inferiores en la categoría de Geografía Física, lo que indica que la corrección favorece a las revistas de ciencias sociales frente a las de ciencia al tener en cuenta el contexto de la cita y dar mayor peso a las citas del campo de las ciencias sociales; así se confirma en la Tabla 1, donde se muestra que los factores de impacto son superiores en la categoría de Geografía Física, mientras que los valores de SNIP son muy parecidos entre la dos categorías.

El índice $\mathrm{H}$ también obtiene unos valores de correlación inferiores a 0,90 en todos los casos, aunque muy próximos a él en la categoría de Geografía y sensiblemente inferiores en la categoría de Geografía Física. Esto puede deberse a que los artículos publicados en Geografía Física tienen un impacto más alto los primeros años, pero los de Geografía tardan más en citarse, ya que este índice refleja el impacto de las revistas y de las citas que reciben a largo plazo, mientras que el resto de indicadores analizan el impacto a más corto plazo.

\subsection{Concordancia entre indicadores en la clasificación de las revistas en cuartiles}

Para estimar el grado de acuerdo en la posición de las revistas en cada indicador, se ha utilizado el coeficiente de concordancia Kappa ponderado. Par ello, se han clasificado las revistas de cada categoría en cuartiles según los valores obtenidos en cada indicador. Los resultados obtenidos se recogen en la Tabla 4.

Como observación general, hay que destacar que el acuerdo entre los indicadores de popularidad en la clasificación de revistas en ambas categorías temáticas es excelente, con valores superiores a 0,800, siendo ligeramente más altos en la categoría de Geografía Física.

El análisis de la concordancia entre los indicadores de popularidad con los de prestigio y el SNIP e índice $\mathrm{H}$, permite afirmar lo siguiente:

- En ningún caso se consigue un acuerdo excelente, salvo la concordancia entre AIS e IPP en la categoría de Geografía. En todos los demás, el valor del índice de Kappa es menor de 0,800.

- Existen diferencias entre las dos categorías temáticas. Mientras que en la categoría de Geografía existe una concordancia buena en todos los casos, con valores superiores a 0,600, en la de Geografía Física existen 10 índices con concordancia moderada $(0,410 \leq 0,600)$ y solo en un caso se obtiene una concordancia débil, entre AIS e índice $\mathrm{H}$. 
- El índice $\mathrm{H}$ es el indicador que obtiene un índice de Kappa más bajo con todos los demás en la categoría de Geografía Física. La razón es que algunas revistas que ocupan posiciones altas con los indicadores de popularidad y de prestigio se caracterizan porque un pequeño número de artículos reciben un gran número de citas y la mayoría de artículos no reciben citas a lo largo del tiempo, viéndose superadas por otras revistas que publican artículos que al principio no reciben muchas citas ninguno de ellos pero a lo largo de los años el conjunto de sus artículos tiene más impacto.

Tabla 4. Concordancia entre los indicadores bibliométricos en las revistas de las categorías JCR Geografía y Geografía Física

\begin{tabular}{|l|c|c|c|c|c|c|c|c|}
\hline \multicolumn{1}{|c|}{ Indicador } & F1 & FI5 & AIS & SJR & FIScopus & SNIP & IPP & Índice H \\
\hline F1 & & 0,834 & 0,757 & 0,768 & 0,831 & 0,621 & 0,801 & 0,684 \\
\hline FI5 & 0,858 & & 0,878 & 0,778 & 0,801 & 0,696 & 0,889 & 0,730 \\
\hline AIS & 0,752 & 0,893 & & 0,744 & 0,701 & 0,663 & 0,812 & 0,786 \\
\hline SIR & 0,716 & 0,752 & 0,716 & & 0,768 & 0,600 & 0,778 & 0,705 \\
\hline FIScopus & 0,893 & 0,858 & 0,752 & 0,752 & & 0,600 & 0,801 & 0,663 \\
\hline SNIP & 0,539 & 0,539 & 0,468 & 0,575 & 0,504 & & 0,736 & 0,684 \\
\hline IPP & 0,858 & 0,893 & 0,787 & 0,787 & 0,893 & 0,575 & & 0,757 \\
\hline Índice H & 0,415 & 0,486 & 0,451 & 0,451 & 0,486 & 0,274 & 0,486 & \\
\hline
\end{tabular}

Leyenda: Geografía: valores por encima de la diagonal; Geografía Física: valores por debajo de la diagonal.

Fuente: elaboración propia a partir de datos del Journal Citation Reports (2016), Scimago Journal Rank (2016) y Journal Metrics (2016)

Las Tablas 5 y 6 presentan información más detallada sobre el rango obtenido por las revistas de las categorías Geografía y Geografía Física, respectivamente, situadas en el primer cuartil del FI en los distintos indicadores. El rango de las revistas en un aspecto muy importante para los investigadores, editores y bibliotecarios, entre otros. Que una revista esté situada en el puesto $n^{\circ} 1$ o en el $15^{\circ}$ es clave, y la pérdida de unas cuantas posiciones puede ser percibida como algo negativo y merma de calidad y de competitividad.

Al comparar los diferentes indicadores, se observa que en las revistas que ocupan los primeros lugares hay un alto grado de coincidencia entre el FI del JCR con el FI de Scopus, FI 5 años, SJR, IPP y AIS; este grado de coincidencia es total en las 4 primeras revistas del Geografía Física. Conforme se va descendiendo en el rango, hay mayor disparidad y la correspondencia es moderada e incluso baja en algunos casos.

Algunas de las revistas situadas en las primeras posiciones del FI aparecen en lugares muy retrasados con el índice $\mathrm{H}$; esto indica que son revistas que a corto plazo reciben un número 
importante de citas mientras que en su trayectoria a largo plazo, se ven superadas por otras revistas (el FI analiza las citas a corto plazo mientras que el índice $\mathrm{H}$ es un reflejo de la influencia de la revista a largo plazo). Es por ello, que el índice $\mathrm{H}$ puede ser un indicador mucho más útil en determinadas revistas y reflejar mejor la influencia de las revistas en la comunidad científica, sobre todo en áreas en las que la vida media citada sea larga, aunque también penalizará a aquellas revistas de áreas con menor número de investigadores.

Los indicadores bibliométricos ofrecen diferente información, como se desprende de las diferencias observadas en la clasificación. Hay que tener presente que algunos analizan el impacto de las citas a corto plazo, y otros a más largo plazo, teniendo en cuenta otros factores como el prestigio de las revistas citantes.

Tabla 5. Revistas de la categoría Geografía situadas en el primer cuartil del FI y su posición en los indicadores bibliométricos

\begin{tabular}{|l|c|c|c|c|c|c|c|c|}
\hline & FI & FI5 & AIS & SJR & FI2SC & SNIP & IPP & H \\
\hline Global Environmental Change-Human and Policy Dimensions & 1 & 1 & 1 & 3 & 1 & 3 & 1 & 2,5 \\
\hline Progress in Human Geography & 2 & 2 & 2 & 1 & 2 & 1 & 2 & 1 \\
\hline Transactions of the Institute of British Geographers & 3 & 4 & 4 & 2 & 3 & 2 & 3 & 8 \\
\hline Landscape and Urban Planning & 4 & 6 & 15 & 14 & 4 & 13 & 4 & 2,5 \\
\hline Economic Geography & 5 & 3 & 3 & 6 & 15 & 7 & 9 & 16 \\
\hline Political Geography & 6 & 9 & 6 & 4,5 & 6 & 5 & 5 & 13,5 \\
\hline Journal of Transport Geography & 7 & 7 & 17 & 8 & 7 & 10 & 8 & 19 \\
\hline Applied Geography & 8,5 & 11 & 23 & 22 & 5 & 11 & 7 & 22 \\
\hline Journal of Economic Geography & 8,5 & 5 & 5 & 4,5 & 10 & 4 & 6 & 13,5 \\
\hline Annals of the Association of American Geographers & 10 & 12 & 10 & 11 & 8 & 15 & 10 & 5 \\
\hline Antipode & 11 & 8 & 7 & 7 & 9 & 6 & 11 & 11,5 \\
\hline Regional Studies & 12 & 16 & 16 & 21 & 20 & 18 & 17 & 6 \\
\hline Geographical Journal & 13 & 19 & 19 & 26 & 12,5 & 29 & 20 & 26 \\
\hline Cultural Geographies & 14 & 22 & 18 & 15 & 19 & 25 & 27 & 36 \\
\hline Population Space and Place & 15 & 23 & 20 & 17 & 18 & 20 & 22 & 28,5 \\
\hline Geoforum & 16 & 14 & 14 & 12 & 12,5 & 23 & 13 & 10 \\
\hline International Journal of Urban and Regional Research & 17 & 13 & 13 & 9 & 14 & 8 & 12 & 11,5 \\
\hline International Journal of Geographical Information Science & 18 & 18 & 30 & 19 & 17 & 14 & 14 & 7 \\
\hline Environment and Planning A & 19 & 15 & 11 & 18 & 21,5 & 26 & 24 & 4 \\
\hline
\end{tabular}

Fuente: elaboración propia a partir de datos del Journal Citation Reports (2016), Scimago Journal Rank (2016) y Journal Metrics (2016)

Tabla 6. Revistas de la categoría Geografía Física situadas en el primer cuartil del FI y su posición en los indicadores bibliométricos

\begin{tabular}{|l|c|c|c|c|c|c|c|c|}
\hline & $\mathrm{FI}$ & $\mathrm{FI} 5$ & $\mathrm{AIS}$ & $\mathrm{SJR}$ & $\mathrm{FI} 2 \mathrm{SC}$ & $\mathrm{SNIP}$ & $\mathrm{IPP}$ & $\mathrm{H}$ \\
\hline Global Ecology and Biogeography & 1 & 1 & 1 & 1 & 1 & 3 & 1 & 5 \\
\hline Cryosphere & 2 & 2 & 2 & 2 & 2 & 12 & 2 & 30 \\
\hline Journal of Biogeography & 3 & 3 & 3 & 3 & 3 & 9 & 4 & 2 \\
\hline Quaternary Science Reviews & 4 & 4 & 4 & 4 & 4 & 13 & 3 & 1 \\
\hline Landscape Ecology & 5 & 7 & 9 & 10 & 7 & 16 & 10 & 7,5 \\
\hline Journal of Quarternary Science & 6 & 17 & 12 & 13 & 11 & 25 & 14 & 14,5 \\
\hline International Journal of Digital Earth & 7 & 18 & 23 & 19 & 12 & 5 & 16 & 41,5 \\
\hline Journal of Glaciology & 8 & 11 & 5 & 5 & 9 & 17 & 11 & 17 \\
\hline ISPRS Journal of Photogrammetry and Remote Sensing & 9 & 5 & 13 & 7 & 5 & 1 & 5 & 14,5 \\
\hline
\end{tabular}


Tabla 6. Continuación

\begin{tabular}{|l|c|c|c|c|c|c|c|c|}
\hline & FI & FI5 & AIS & SJR & FI2SC & SNIP & IPP & H \\
\hline Landscape and Urban Planning & 10 & 9 & 18 & 14 & 8 & 7 & 8 & 6 \\
\hline $\begin{array}{l}\text { IEEE Journal of Selected Topics in Applied Earth Observations and } \\
\text { Remote Sensing }\end{array}$ & 11 & 12 & 22 & 8 & 6 & 2 & 9 & 32,5 \\
\hline
\end{tabular}

Fuente: elaboración propia a partir de datos del Journal Citation Reports (2016), Scimago Journal Rank (2016) y Journal Metrics (2016)

Al examinar los rangos y sus discrepancias entre los indicadores de popularidad y de prestigio, nos encontramos con revistas que pueden pertenecer a estas cuatro categorías (Franceschet, 2010):

- Revistas con alta popularidad y prestigio: Son revistas muy citadas y las citas proceden de revistas prestigiosas. Por ejemplo, Global Environmental Change-Human and Policy Dimensions, Progress in Human Geography y Transactions of the Institute of British Geographers en la categoría de Geografía, y Global Ecology and Biogeography, Cryosphere y Journal of Biogeography de Geografía Física.

- Revistas con alta popularidad y bajo prestigio: Son revistas con tasa alta de citas pero procedentes de revistas de bajo prestigio. Por ejemplo, Applied Geography y Geographical Journal en la categoría de Geografía, o International Journal of Digital Earth en Geografía Física.

- Revistas con baja popularidad y alto prestigio: Estas revistas reciben relativamente pocas citas en comparación con el número de artículos que publican, pero sus citas provienen de revistas muy prestigiosas. Por ejemplo, Cambridge Journal of Regions Economy and Society de la categoría Geografía (no aparece en la tabla) y Progress in Physical Geography o Geoinformatica de la categoría Geografía Física (ambas no aparecen en las tablas).

- Revistas con baja popularidad y prestigio: Revistas poco citadas y las citas las reciben de revistas poco importantes. Por ejemplo, Mitteilungen Der Osterreichischen Geographischen Gesellschafta de la categoría de Geografía o Acta Geographica Slovenica de la categoría de Geografía Física.

A partir de los datos de la tabla, resulta conveniente distinguir las revistas que son muy populares en dos tipos. Aquellas que reciben muchas citas pero se distribuyen entre varios artículos, y las que reciben muchas citas pero están concentradas en muy pocos artículos. La primera situación corresponde a revistas que ocupan primeras posiciones en los indicadores de popularidad y en el índice H, como Progress in Human Geography y Global Ecology and Biogeograpcy. La segunda situación se produce en revistas que ocupan primeros lugares en los indicadores de popularidad y más retrasados en el índice $H$, como la revista Cryosphere, clasificada en segundo lugar entre los indicadores de popularidad y de prestigio, pero en la posición 30 según el índice $\mathrm{H}$.

A pesar de que existe una correlación alta entre algunos indicadores, especialmente entre los de mismo tipo, el rango de las revistas varía sensiblemente entre ellos, situación que coincide con los 
resultados obtenidos por Gray y Hodkinson (2008) en revistas de áreas próximas a Geografía como Ecología y Ciencias Ambientales o con los de Elkins (2010) en su análisis de todas las revistas del JCR en el año 2007.

Se ha comprobado que el FI y el FI5 llevan al mismo rango de las revistas en categorías que contienen más de 140 revistas, especialmente en aquellas situadas al principio y al final del rango, por lo que el FI5 no añade nada nuevo uno sobre el otro. Sin embargo, los rangos de revistas específicas pueden cambiar, y la impresión es que en algunos campos un periodo de dos años es demasiado corto para evaluar las revistas, por lo que hay que considerar cada situación específica (The Stimulate 9 Group, 2009).

Por lo tanto, los investigadores deberían considerar otros indicadores además del FI a la hora de elegir en qué revistas publicar, ya que puede ser más apropiado un indicador que tenga en cuenta un periodo de citación de 5 años si la revista pertenece a un área donde la citación se produce a largo plazo.

\subsection{Limitaciones}

Las categorías temáticas de WoS son ampliamente utilizadas para la normalización en prácticas bibliométricas. Sin embargo, poco se sabe sobre la precisión de este sistema de clasificación, y Pudovkin y Garfield (2002) ya avisaban de que la asignación de las revistas a estas categorías es subjetiva y utiliza métodos heurísticos, como el algoritmo de Hayne-Coulson, que no se ha publicado nunca, especificado como "el número de citas que cada revista recibe de diferentes revistas núcleo especializadas se obtiene anualmente por una rutina informática utilizada para crear la bases de datos JCR" (Pudovkin y Fuseler, 1995).

Más recientemente, Leydesdorff y Bornmann (2015), en un estudio sobre dos áreas de investigación, "Library and Information Science" y "Science \& Technology Studies", concluían que la agrupación de revistas en categorías puede no ser suficiente precisa y afectar a la calidad de la evaluación. Wang y Waltman (2016), al analizar la precisión de los sistemas de clasificación de revistas en WoS y Scopus mediante las relaciones directas de citas entre revistas y categorías, sugieren que WoS y Scopus son demasiado indulgentes al asignar revistas a categorías, si bien WoS presenta mejores resultados de clasificación.

A pesar de que la exactitud de los sistemas de clasificación de revistas en WoS y Scopus ha sido un tema de interés y de estudio para mejorar estos sistemas, actualmente se siguen utilizando ya que acudir a expertos para clasificar las revistas manualmente en categorías, sería inviable por el gran número que sería necesario ya que ninguno tendría conocimiento suficiente para clasificar las revistas en todas las disciplinas científicas. 
Se han utilizado indicadores que emplean datos procedentes de dos bases de datos diferentes, WoS y Scopus. Para evitar un posible sesgo producido por esta causa, se han seleccionado únicamente aquellas revistas que coinciden en ambas bases de datos, por lo que el número de artículos es el mismo, aunque el número de citas que reciben depende de cada base de datos. Existen estudios en la bibliografía en los que se ha evidenciado que los indicadores son independientes de la base de datos (Archambault et al., 2009).

\section{Conclusiones}

Los temas de estudio en el área científica de Geografía son de carácter multidisciplinar, por lo que parte de sus investigaciones se publican en revistas pertenecientes a otros campos de conocimiento y, por tanto, clasificadas en otras categorías de JCR. Esta dispersión puede explicar, en parte, que los factores de impacto de las disciplina sean más bajos que los de otras áreas científicas más especializadas.

Del estudio desarrollado en este trabajo se extraen algunas generalizaciones sobre el impacto e influencia de las revistas de Geografía de las que se derivan recomendaciones prácticas a tener en cuenta en la evaluación de la actividad científica de los geógrafos:

a) El impacto de las revistas de la categoría de Geografía Física es significativamente mayor que el de las revistas de la categoría de Geografía. Esta diferencia no se produce con los indicadores de prestigio.

b) El grado de correlación encontrado entre todos los indicadores de popularidad y de prestigio es muy elevado. Esta convergencia indica que miden características similares de las revistas y que los factores diferenciadores que utilizan en sus fórmulas, relacionados con el número de años o ponderación de las revistas según su prestigio influyen, muy levemente en los resultados.

Los indicadores SNIP e índice $H$ son los que presentan menor grado de correlación con los indicadores de popularidad y de prestigio y, por tanto, indica que miden dimensiones diferentes y aportan información complementaria.

c) La clasificación de revistas en cuartiles muestra un elevado grado de concordancia entre los indicadores de popularidad, lo que refuerza que la ventana temporal o la base de datos utilizada son factores que no influyen de manera importante en el rango de las revistas. La concordancia es menor, aún siendo moderada, con los indicadores de prestigio, y se obtienen los peores resultados con los indicadores SNIP e índice $\mathrm{H}$.

d) Las revistas que ocupan los primeros puestos en las clasificaciones de los indicadores de popularidad y de prestigio son las mismas. A partir del cuarto puesto en la categoría de Geografía y del quinto en Geografía Física se producen discrepancias importantes que, en 
determinados casos, afecta a la clasificación de las revistas en cuartiles diferentes según los indicadores utilizados.

e) El indicador SNIP y el índice H presentan mayores diferencias en el ranquin, proporcionando este último información de gran utilidad al permitir averiguar qué revistas tienen mucho impacto pero debido solamente a muy pocos artículos.

El comportamiento de los indicadores analizados es muy similar en las dos categorías temáticas de la disciplina de Geografía. Posibles diferencias entre indicadores (o las similitudes entre los indicadores) se pueden derivar del uso de bases de datos diferentes como fuentes de citas, WoS y Scopus, así como de la diferente metodología en la estimación de estos indicadores.

Todas las revistas publican en sus sitios web parámetros relacionados con su impacto, popularidad, visibilidad en bases de datos y su situación en cuartiles. La profusión de indicadores hace recomendable que los investigadores conozcan el significado de los más utilizados para seleccionar aquellas revistas en que la difusión de sus trabajos vaya a tener mayor repercusión.

La posición de una revista en un determinado cuartil, requisito utilizado en el sistema de evaluación de la actividad científica del personal docente e investigador en España, puede variar según el indicador que se utilice y afectar al resultado de dicha evaluación. Por lo tanto, a la hora de evaluar la calidad de las revistas de geografía, es necesario y recomendable utilizar varios indicadores que ofrezcan información complementaria y faciliten una valoración más objetiva de su importancia y repercusión en la comunidad científica. El índice $H$ es un complemento útil de los factores de impacto; en primer lugar, por su robustez, ya que es insensible a un número muy elevado de artículos no citados y a unos pocos artículos muy citados; en segundo lugar, combina tanto la cantidad (número de publicaciones) como la calidad (medida en número de citas), y por tanto, reduce la sobrevaloración de revistas pequeñas pero con muchos artículos de revisión.

Se ha señalado en la bibliografía que la calidad de una revista, medida a través de indicadores de popularidad y prestigio, es un concepto multidimensional en el que pueden intervenir varios factores como hábitos de citación, tamaño de la comunidad científica, tiempo, etc. La altísima correlación encontrada entre algunos indicadores sugiere que recogen la misma información y que las diferencias en sus fórmulas no afectan al rango de las revistas, por lo que habría que plantearse si son necesarios diferentes indicadores que miden realmente lo mismo y que no añaden información nueva. 


\section{Bibliografía}

Althouse, B. M., West, J. D., Bergstrom, C. T., y Bergstrom, T. (2009). Differences in impact factor across fields and over time. Journal of the American Society for Information Science and Technology, 60(1), 27-34. doi: 10.1002/asi.20936

Archambault, E., y Lariviere, V. (2009). History of the journal impact factor: Contingencies and consequences. Scientometrics, 79(3), 635-649.

Archambault, E., Campbell, D., Gingras, Y., y Larivière, V. (2009). Comparing bibliometric statistics obtained from the web of science and Scopus. Journal of the American Society for Information Science and Technology, 60(7), 1320-1326. doi: 10.1002/asi.21062.

Barriocanal, C., y Copete, J. L. (2005). La relevancia de las revistas de geografía física a partir de su representación en el Journal Citation Reports. Revista de Geografía, 4, 95-104.

Bar-llan, J. (2012). Journal report card. Scientometrics, 92(2), 249-261.

Bergstrom, C. (2007). Eigenfactor. Measuring the value and prestige of scholarly journals. College and Research Library News, 68(5), 314-316.

Bergstrom, C. T., West, J. D., y Wiseman, M. (2008). The Eigenfactor metrics. The Journal of Neuroscience, 28(45), 11433-11434. doi: 10.1523/JNEUROSCI.0003-08.2008

Bodman, A. R. (1991). Weavers of influence: The structure of contemporary geographic research. Transactions of the Institute of British Geographers, 16(1), 21-37.

Bollen, J., Rodriquez, M. A., y Van De Sompel, H. (2006). Journal status. Scientometrics, 69(3), 669-687. doi: 10.1007/s11192-006-0176-z

Bosman, J. (2009). The changing position of society journal in geography. Journal of Economic and Social Geography, 100(1), 20-32. doi: 10.1111/j.1467-9663.2009.00511.x

Braun, T., Glanzel, W., y Shubert, A. (2006). A Hirsch-type index for journals. Scientometrics, 69(1), 169-173.

Callaham, M., Weber, E., y Wears, R. (2001). Citation characteristics of research published in Emergency Medicine versus other scientific journals. Annals of Emergency Medicine, 38(5), 513517.

Chou, P. (2013). An Exploratory Study of Database Evaluation Metrics: Comparison between IF and SJR. International Journal of Computer and Information Technology, 2(1), 25-28.

Cicchetti, D., y Allison, T. (1971). A new procedure for assessing reliability of scoring EEG sleep recordings. American Journal of EEG Technology, 11(3), 101-110. doi: 10.1080/00029238.1971.11080840 
Cohen, J. (1968). Weighted kappa: nominal scale agreement with provisions for scaled disagreement or partial credit. Psychological Bulletin, 70(4), 213-20.

Elkins, M. R., Maher, C. G., Herbert, R. D., Moseley, A. M., y Sherrington, C. (2010). Correlation between the Journal Impact Factor and three other journal citation indices. Scientometrics, 85(1), 81 93.

Elsevier, B. V. (2015). Journal metrics. Recuperado el 20 de marzo de 2016, de http://www.journalmetrics.com/

Franceschet, M. (2010). The difference between popularity and prestige in the sciences and in the social science: A bibliometric analysis. Journal of Informetrics, 4(1), 55-63.

García-Pachón, E., y Arencibia-Jorge, R. (2014). Comparación del factor de impacto y el índice Scimago Journal Rank en las revistas del sistema respiratorio. Archivos de Bronconeumología, 50(7), 308-309.

García Ruiz, J. M. (2008). Las citas internacionales de las revistas españolas de Geografía. Boletín de la Asociación de Geógrafos Españoles, 46, 207-225.

Garfield, E. (2006). The history and meaning of the journal impact factor. Journal of the American Medical Association, 295(1), 90-93.

Glanzel, W. y Moed, H. (2002). Journal impact measures in bibliometric research. Scientometrics, $53(2), 171-193$.

González-Pereira, B., Guerrero-Bote, V. P., y Moya-Anegón, F. (2010). A new approach to the metric of journals scientific prestige: The SJR indicator. Journal of Informetrics, 4(3), 379-391. doil:10.1016/j.joi.2010.03.002

Gray, E., y Hodkinson, S. Z. (2008). Comparison of Journals Citation Reports and Scopus Impact Factors for Ecology and Environmental Science Journals. En Issues in Science and Technology Librarianship, n. ${ }^{\circ}$ 54. doi:10.5062/F4FF3Q9G

Hirsch, J. E. (2005). An index to quantify an individual's scientific research output. Proceedings of the National Academy of Sciences of the United States of America, 102(46), 16569-16572. doi:10.1073/pnas.0507655102

Huang, D. W. (2016). Positive correlation between quality and quantity in academic journals. Journal of Informetrics, 10(2), 329-335.

Laffan, S. W. (2010). The citation relationships between journals of geography and cognate disciplines. Geographical Research, 48(2), 166-180.

Landis, J. R., y Koch, G. G. (1977). The measurement of observer agreement for categorical data. Biometrics, 33(1), 159-174. 
Leydesdorff, L. (2008). Caveats for the use of citation indicators in research and journal evaluations. Journal of the American Society for Information Science and Technology, 59(2), 278-287.

Leydesdorff, L., y Bornmann, L. (2015). The operationalization of "fields" as WoS subject categories (WCs) in evaluative bibliometrics: The cases of "Library and Information Science" and "Science \& Technology Studies". Journal of the American Society for Information Science and Technology, 67,(3), 707-714.

Moed, H. F. (2010). Measuring contextual citation impact of scientific journals. Journal of Informetrics, 4(3), 265-277. doi: 10.1016/j.joi.2010.01.002

Mutz, R., y Daniel, H. D. (2012). Skewed citation distributions and bias factors: Solutions to two core problems with the journal impact factor. Journal of Informetrics, 6(2), 169-176.

Nederhoff, A. J. (2006). Bibliometric monitoring of research performance in the Social Sciences and the Humanities: A review. Scientometrics, 66(1), 81-100.

Pendlebury, D. A., y Adams, J. (2009). The use and misuse of journal metrics and other citation indicators. Archivum of Immunologiae et Therapiae Experimentalis, 57(1), 1-11.

Pudovkin, A. I., y Fuseler, E. A. (1995). Indices of journal citation relatedness and citation relationships among aquatic biology journals. Scientometrics, 32(3), 227-236.

Pudovkin, A. I., y Garfield, E. (2002). Algoritmic procedure for finding semantically related journals». Journal of the American Society for Information Science and Technology, 53(13), 11131119.

Richards, K., Batty, M., Edwards, K., Findlay, A., Foody, G., Frostick, L. et al. (2009). The nature of publishing and assessment in Geography and Environmental Studies: evidence from Research Assessment Exercise 2008. Areas, 41(3), 231-243.

Rohli, R. V., y Kam-Biu, L. (2008). Citation of research in journals of interest to applied geographers. Applied Geography, 28, 151-167.

Scarletto, E. A. (2014). Mapping the literature of GIS. College and Research Libraries, 75(2), 179181.

Seglen, P. O. (1997). Why the impact factor of journals should not be used for evaluating research. British Medical Journal, 314(7079), 498-502.

Sellers, S. L., Mathiesen, S. G., Perry, R., y Smith, T. (2004). Evaluation of social work journal quality: Citation versus reputation approaches. Journal of Social Work Education, 40(1), 143-160.

Setti, G. (2013). Bibliometrics indicators: Why do we need more than one? IEEE Access, 1, 232-246. 
The Stimulate 9 Group. (2009). The 5-year synchronous impact factor for large Journal Citation Reports (JCR) subject areas. Cybermetrics, 13(1), 11-14.

Vanclay, J. K. (2012). Impact factor: outdated artefact or stepping-stone to journal certification? Scientometrics, 92(2), 211-238. doi: 10.1007/s11192-011-0561-0

Waltman, L. (2016). A review of the literature on citation impact indicators. Journal of Informetrics, 10(2), 365-391.

Wang, Q., y Waltman, L. (2016). Large-scale analysis of the accuracy of the journal classification systems of Web of Science and Scopus. Journal of Informetrics, 10(2), 347-364.

Yin, C. Y. (2011). Do impact factor, h-index and Eigenfactor of chemical engineering journals correlate well with each other and indicate the journals' influence and prestige? Current Science, 100(5), 648-653. 